\title{
DEVELOPMENT AND VALIDATION OF A FINITE ELEMENT MODEL TO PREDICT PATELLO-FEMORAL WEAR IN TKA
}

\author{
Silvia Pianigiani (1), Yan Chevalier (2), Luc Labey (2), Walter Pascale (1), Amir \\ Kamali (3), Bernardo Innocenti (2)
}

\author{
1. I.R.C.C.S Istituto Ortopedico Galeazzi, Milano, Italy; 2. European Center for Knee \\ Research, Smith\&Nephew, Leuven, Belgium; 3. Implant Development Centre (IDC), \\ Smith and Nephew Orthopaedics Ltd, Leamington Spa, UK
}

\section{Introduction}

Experimental patello-femoral (PF) and femoraltibio (FT) wear tests can be used to quantify wear in an implant but these procedures are expensive and time consuming. Therefore, a validated numerical model could be useful to predict wear in less time with less cost. For these reasons, the aim of this study was to develop and validate a numerical methodology to predict PF wear in TKA. Initially, the wear model was calibrated using the results of an experimental roll-on-plane wear test. The developed wear model was applied to predict PF wear and validated comparing the numerical predicted wear with experimental wear results.

\section{Methods}

Wear model

The adhesive/abrasive wear behavior was determined using the Archard model [Archard, 1953] with the Sarkar modification [Sarkar, 1980], in which linear wear $\mathrm{h}$ is determined as:

$$
\mathrm{h}=\mathrm{k}_{\mathrm{w}} \text { p s } \sqrt{ }\left(1+3 \mu^{2}\right)
$$

in which: $\mathrm{k}_{\mathrm{w}}=$ wear factor, $\mathrm{p}=$ contact pressure, $\mathrm{s}=$ sliding distance, $\mu$ friction coefficient.

Roll-on-plane calibration

Three blocks of UHMWPE (GUR 1020) underwent a $6 \times 10^{6}$ cycles experimental roll-on-plane wear test (Fig. 1a). The full experimental test was reproduced by finite element analysis (FEA) (Fig. 1b) and the wear model was calibrated, adjusting $\mathrm{k}_{\mathrm{w}}$, during the simulation as illustrated in Figure 2. The geometry of the block was updated every 500.000 cycles.

Patello-femoral wear prediction

Three patellar and femoral components were tested for $2 \times 10^{6}$ cycles in a wear simulator (Fig. 1c) [Vanbiervliet, 2011]. The full experimental test was numerically analyzed in a FEA model (Fig. 1d). The PF wear was predicted using the calibrated wear model (Fig. 2). Experimental wear data and numerical wear prediction were finally compared.

\section{Results}

Roll-on-plane

Using a $\mathrm{k}_{\mathrm{w}}=1.83 \times 10-8 \mathrm{~mm}^{3} / \mathrm{Nm}$, the results of the numerical simulation show a linear wear of 0.127 $\mathrm{mm}$, very close to the linear wear measured experimentally $(0.125 \mathrm{~mm}, \mathrm{SD}=0.01 \mathrm{~mm})$.

Patello-femoral

The numerical model results show a total volume wear of $0.38997 \mathrm{~mm}^{3}$ after $2 \times 10^{6}$ cycles while the mean wear volume measured experimentally for the same number of cycles for three samples, is $0.37660 \pm 0.326$ [Vanbiervliet, 2011].
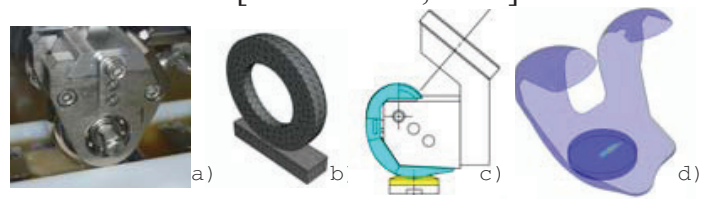

Figure 1: a) detail of roll-on-plane experimental machine; b) numerical roll-on-plane simulation; c) PF experimental model (scheme); d) PF numerical model

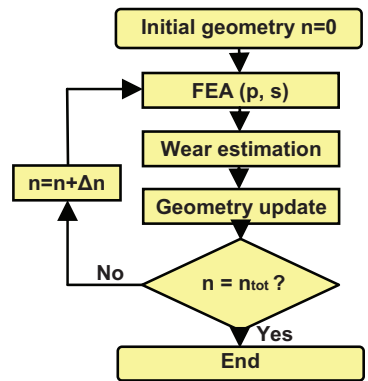

Figure 2: flow chart of the wear estimation in the finite element model

\section{Discussion}

In this study a wear model was developed and calibrated comparing an experimental and a numerical roll on plane test. The wear model was later validated comparing the wear predicted by the numerical model and from experimental test of $2 \times 10^{6}$ cycles of walking. The validated wear model can be used to predict wear between PF and FT articulations for several configurations to predict a TKA long-term performance for a specific patient and to optimize and improve implants designs.

\section{References}

Archard, J. Appl. Phys. 24 (1953) 981.

Sarkar, London: Academic Press, 1980.

Vanbiervliet, JBJS Br, 1348-54, 93-B(10), 2011. 